Ellen Markgraf

\title{
Die Wandlung des Künstlerateliers - VON DEM ORT DER INSZENIERUNG ZU DER INSZENIERUNG DES ORTES ${ }^{1}$
}

Den allgemeinen Zusammenhang dieser Gedanken bildet die Frage nach der Existenz und der Präsentation von Künstlerateliers in ihrer historischen Entwicklung; außerdem richtet sich der Focus in diesem Beitrag auf einen relevanten Bestandteil, den Tisch, in seinen verschiedenen tragenden Rollen.

Dieser alltägliche Gegenstand erscheint als ein ausgesprochen vielschichtiges Motiv in den Werken der Bildenden Kunst. Betrachtet man die Entwicklung seiner Darstellung, so lässt sich ein klares Fazit ziehen: Der Tisch wird von einem Objekt zu einem Subjekt - das Leben an einem Tisch entwickelt sich zu einem Tisch-Dasein. Galt er bis in die Anfänge des 20. Jahrhunderts und teilweise auch noch darüber hinaus als ein Objekt, an dem unzählige Aspekte des Lebens geschehen, so zeigt sich im fortschreitenden 20 . und beginnenden 21. Jahrhundert seine alleinige Existenz - in dem Sinne wird er zu einem Stellvertreter des Menschen - gleichsam in Abwesenheit Anwesender. Dieses Faktum kann als Spiegelung des Menschen und seiner Lebensweisen gesehen werden. Der Tisch wird so zu einer Metapher für den Menschen selbst.

Der formale Träger der Inhalte ist ein wesentliches Detail im Kontext der Frage nach der möglichen Wandlung des Ateliers, die in der Überschrift des Beitrages bereits deutlich wird. Kann das inszenierte Atelier als Metapher für die Kunstschaffenden gelesen werden?

Das Atelier in seiner Existenz und Wandlung einerseits, der Tisch als definierte Metapher andererseits sind die wesentlichen Komponenten des vorliegenden Aufsatzes.

1 Dieser Text ist eine veränderte und erweiterte Erarbeitung zu der übergeordneten Thematik. Ausgangspunkt ist ein Vortrag mit dem Thema „Der Tisch als Metapher für ... - Die ,Große Tischruine“ von Dieter Roth“ im Rahmen des Symposiums „Atelier und Dichterzimmer in neuen Medienwelten“ im Mai 2004. 


\section{Das Atelier in der Tradition ...}

Die traditionelle Interpretation eines Künstlerateliers zeigt sich in dem Gemälde „Galeriedarstellung mit Pictura - Allegorie“ von Cornelis de Bailleur (um 1640). Zu sehen ist eine Atelier- und Werkstattsituation - und damit nicht genug, denn im Grunde erscheint der Innenraum wie eine Ausstellung im Sinne einer Bild-Präsentation (Abb. 1).

Das Interieur ist gefüllt mit mehreren Tischen und vielen Gemälden; der Betrachtende entdeckt rechts an der Seite des Bildes die Werkstatt und außerdem ist die Anwesenheit von drei Personen und zahlreichen Gegenständen, unter vielen anderen auch Malutensilien, auf dem

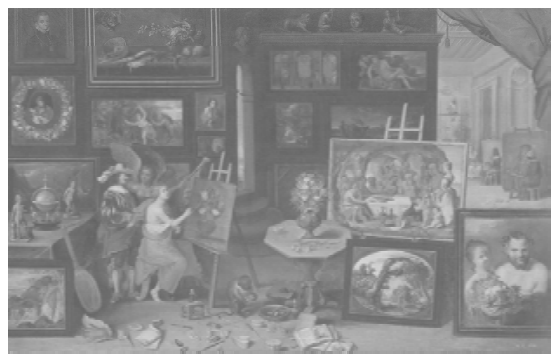

Abb. 1: Galeriedarstellung mit

Pictura - Allegorie" von

Cornelis de Bailleur (um 1640)

Fußboden zu konstatieren. Die Tische zeigen sich in diesem Kontext nicht als Handlungsträger, sondern als Flächen zur Ablage; für den Gegenstand des aktuellen Gemäldes, den Blumenstrauß und für die Welt des künstlerisch Tätigen sind überdies relevante Objekte zu entdecken. Die Tische, wichtige Requisiten, sind unterschiedlich groß und sie sind ganz differenziert in ihrer Art. Ihre Funktion als Stellfläche ist aus der Gattung Stillleben ja hinlänglich bekannt.

Interessant ist es, festzustellen, dass die in dem Raum ausgestellten Bilder einen genauen Einblick geben in die unterschiedlichen Sujets der Malerei in der damaligen Zeit: Mythologie, Landschaft, das bereits erwähnte Stillleben und Porträtdarstellungen. So zeigen sich Aspekte von Welt einerseits und Aspekte aus dem künstlerischen Mikrokosmos der damaligen Zeit andererseits. Die Bildwelt als Weltbild. „Das Gemälde gewährt uns also offenbar Einblick in eine Künstlerwerkstatt, die sich durch die reiche Sammlung an Kunstwerken und Naturalien als wahrer Mikrokosmos darstellt."

2 Justus Lange: „Galeriedarstellung mit Pictura Allegorie“, in: Pan und Syrinx. Pan und Syrinx. Eine erotische Jagd. Peter Paul Rubens, Pieter Brueghel und ihre Zeitgenossen [Ausstell. Kat.], Kassel, Frankfurt/M. 2004, S. 113. 
Die Allegorie der Malerei, die Malerin in diesem Fall, sitzt an der Staffelei; ein Mann und die Allegorie der Fama stehen seitlich in unmittelbarer Nähe. Es existieren Vermutungen, dass es sich bei dem abgebildeten Atelier um das von Peter Paul Rubens handelt; nicht zuletzt die Darstellung von ,Pan und Syrinx “ an der Wand im Hintergrund ist ein Werk von ihm in Zusammenarbeit mit Jan Brueghel d. Ä.; und so könnte der Mann an der Seite der Malerei der Künstler selbst sein. Die Informationen über die Gegebenheiten in einem Atelier und die Repräsentation des Künstlers, der Künstlerin im Kontext des Werkes sind in dieser Darstellung relevant. Die künstlerische Wirkungsstätte wird in ihren

Abb. 2: „Las Meninas“ von Diego Velázquez

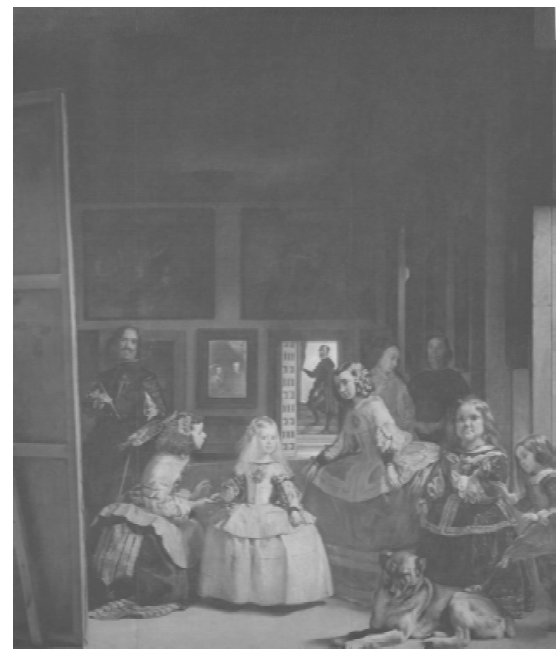

gesamten Facetten in dieser malerischen Abbildung inszeniert. Mit dem Begriff der Inszenierung ist auch das Gemälde ,L Las Meninas “von Diego Velázquez (Abb. 2) zu belegen. Hier erscheint der Künstler ebenfalls und eindeutig selbst und in repräsentativem Gestus. Vor der Leinwand stehend, in entsprechender Haltung, dokumentiert er seine Position als Hofmaler. Den vielen Rätseln um dieses Bild, beispielsweise der Frage nach der Bedeutung des Spiegels an der Rückwand etc., ist hier nicht weiter nachzugehen. Relevant ist der Aspekt, dass die Atelier-Situation in den höfischen Kontext verlagert ist. Auch die, zwar überwiegend im Dunkeln liegende, Bildersammlung an den Wänden unterstützt das Ambiente und verweist auf das Bild von Cornelis de Bailleur (Abb.1).

Der Künstler malt ,vor Ort' des Geschehens und des Auftrages, und vermutlich stehen die Modelle für diese Komposition, die auf der sichtbaren Leinwand entsteht, außerhalb des Bildes. Die Komposition 
lenkt die Blicke der Betrachtenden zunächst auf die Infantin und das Hofpersonal; und dann in einer entsprechenden Blickachse mit dem Mann, der den Raum zu verlassen scheint, ist der Künstler zu entdecken und von dem Moment an unübersehbar.

Geht man noch einige Jahrzehnte, im Grunde Jahrhunderte zurück, so zeigt sich Jan van Eyck als Künstler im Spiegel an der Rückwand in dem Gemälde ,Die Hochzeit der Arnolfini “. Dramaturgisch sind diese beiden Darstellungen hoch interessant - von den Anfängen des Künstler-Selbstporträts bis hin zu der narzisstisch anmutenden Interpretation des spanischen Malers sei hier der Bogen geschlagen.

Geht es um Inszenierung, um Dramaturgie, darf auch der Maler Jan Vermeer nicht außer Acht gelassen werden. Sein einmaliges Konzept, in ein und demselben Raum immer wieder andere Begebenheiten zu inszenieren - vergleichbar einem Bühnenbild auf dem sich seine Protagonisten und vor allem Protagonistinnen zeigen - und malerisch abzubilden, steht beispielhaft für das Atelier in der Tradition, für die Inszenierung des Ortes, an dem der Künstler arbeitet. Wirkungsstätte und Ereignisstätte sind in diesem Kontext identisch. Im Unterschied zu Diego Velázquez zeigt er sich selbst in dem Gemälde „Allegorie der Malerei “, jedoch von hinten. Im Anschluss an die Aspekte der unter anderen barocken „Produktionsstätte“ richtet sich der Focus auf ein gleichsam zeitgenössisches Atelier.

\section{Die „Große Tischruine“ von Dieter Roth}

In diesem Abschnitt stellt sich die Frage nach einem Atelier im Spannungsfeld zwischen Wirkungsstätte und Ausstellungsort. Im Gegensatz zu dem wohlgeordneten Mikrokosmos in dem barocken Bild (s. Abb. 1) gestaltet sich die „Große Tischruine“"von Dieter Roth als wahre Collage mit einer unüberschaubaren Anzahl von Dingen, die in der Kunst, für und mit der Kunst eine Rolle spielen (Abb. 3). Immerhin beträgt die Größe dieser Installation ca. 12x6 m. Entstanden im Atelier und in der Folge ausgestellt, wurde diese Installation immer umfangreicher. Interessant ist der Aspekt, dass das Atelier, das gleichsam „mobile Atelier“" selbst zu dem Gegenstand einer Ausstellung wird.

In dem Gemälde von de Bailleur (vgl. Abb. 1) geht es um die Malerei, die Entstehung und die Präsentation von Gemälden - der Künstler, die Künstlerin waren Maler, Malerin, Bildhauer, Bildhauerin - sieht man jetzt einmal von den genial mehrfach Begabten wie beispielsweise Michelangelo ab. Das Bild des Künstlers wandelt sich im Laufe der Jahrhunderte, und es entsteht, entsprechend dazu die wesentlich vielfältigeren künstlerischen Medien, ein anderes Atelier - der Tisch 
als Metapher für die manuelle künstlerische Arbeitsweise, der Tisch als Metapher für die geistige Produktion, den Filmschnitt, die Filmprojektion etc. Anstelle der Leinwand, anstelle des zu bearbeitenden Steins steht - auf einem Tisch - der Computer als stellvertretendes Element der so genannten Neuen Medien.

Abb. 3: ",Große Tischruine“ von Dieter Roth

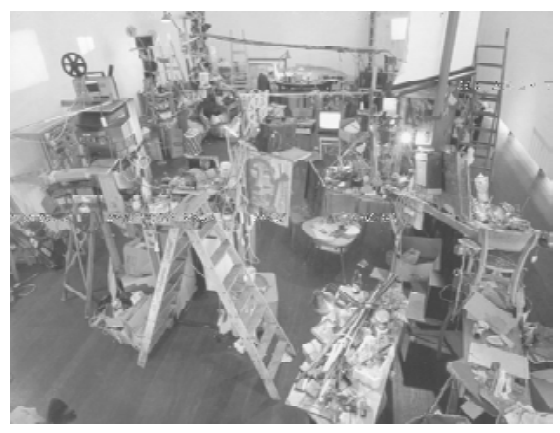

„Auf meinem Ateliertisch an der Danneckerstrasse 32 in Stuttgart fing ich an, 1978/79, ein Bild zu malen, worein ich Kassetten-Aufnahmegeräte baute. Auf diesen Geräten nahm ich die Geräusche, um mich herum, während des Malens auf. Plattenspieler-Musik, eigene Körpertöne u. Ähnliches. Zu jener Zeit hatte ich keine Geduld, irgendetwas malend darzustellen, und besonders auf diesem Gemälde gelang mir nur Geschmier. Am Anfang malte ich auf ein grobes, leichtes Holzgestell aus Latten, das ich auf den Tisch gelegt hatte. (- die Wiederholung der Tischplatte -) Später, um es bequemer zu haben, stellte ich das Brett, mit dem ersten Aufnahmegerät darauf, schräg. Es kam mir bald wie ein Armaturenbrett vor, - nachdem ich ein zweites Aufnahmegerät angebracht hatte, womit ich nicht nur die jeweiligen entstehenden Geräusche aufnahm, sondern auch das auf dem ersten Gerät schon registrierte. “3

Die hier beschriebene Vorgehensweise des Künstlers beschreibt den Kern der „Tischruine“, die er in späteren Jahren unter anderen mit seinem Sohn Björn immer wieder erweitert und ergänzt hat. Dieses Wachstum und Wuchern spiegelt den Schöpfungsprozess bei Roth und begleitet seinen gesamten Lebensweg bis zu seinem Tod (1998). So entsteht eine Schnittstelle zwischen der Schöpfung an sich und den sie spiegelnden und reflektierenden Prozessen. Der Tisch ist Teil des Arbeitsprozesses und wird dann zu dem Bild-Grund, zu der Grundlage der Komposition, zu dem Grund der Komposition.

3 Dieter Roth, in: Das XX. Jahrhundert. Ein Jahrhundert Kunst in Deutschland. [Ausstell. Kat.], Berlin 1999, S. 610. 
Parallel zu dem Gedanken, die die Bilderarbeitung begleitenden Geräusche aufzunehmen, existieren ja auch Filme, ,Ein Tagebuch ' beispielsweise, die Szenen aus dem Leben von Dieter Roth festhalten - oder seiner Existenz nachhaltig immer wieder ein Stück Lebendigkeit verleihen. Sowohl die ,, Große Tischruine “, als auch die Filme waren auf der Documenta11, 2002 im Fridericianum ausgestellt. Anders als im Kontext der Ausstellung in Berlin „Das XX. Jahrhundert in Deutschland“

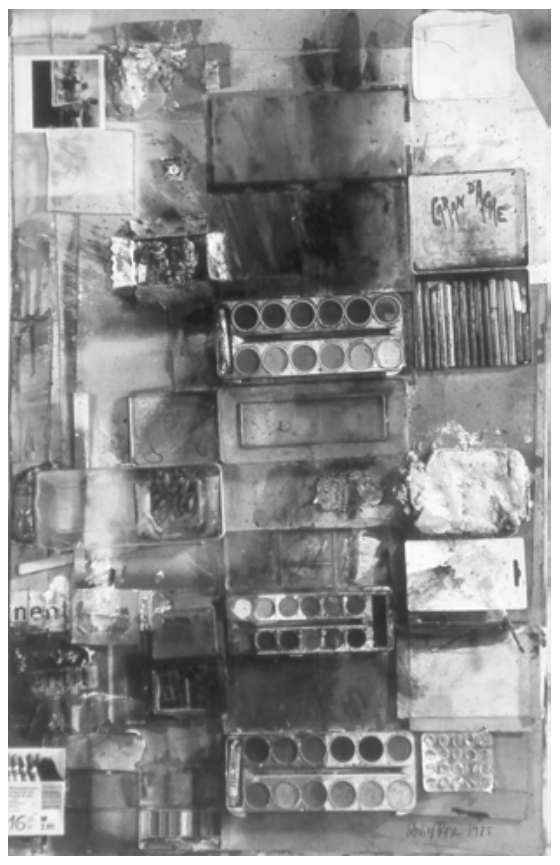

Abb. 4: Dieter Roth „Ohne Titel“

durften sich die Betrachtenden in Kassel nicht in der gesamten Installation bewegen. Es gab eine klare Absperrung. Auch wenn solche Maßnahmen verständlich sind, so fiel jedoch ein entscheidender Teil der Rezeption, das gleichsam haptische Begreifen, weg.

Der Tisch, die Tischplatte wird zum Träger wichtiger Dinge, im Falle Roths unter anderem von Malutensilien und generell Dingen, die in seinem Leben und künstlerischen Werk unterschiedliche Rollen gespielt haben. Der Tisch, im Grunde Träger, wird so selbst zu einem Teil der künstlerischen Installation, zu einem Artefakt.

Vergleichbar mit Aspekten der „Großen Tischruine“ zeigen sich im Werk von Dieter Roth bearbeitete und als Bildwände ausgestellte Tischplatten. In der jüngsten Ausstellung zu dem Werk von Roth in Köln war zwar nicht die „Große Tischruine“ zu sehen, aber dafür andere 
Elemente, die die angesprochene Arbeitsweise spiegeln. So genannte Matten, auf einem Tisch liegend bearbeitet und dann - im wahrsten Sinne des Wortes - als Tafel-Bild aufgehängt und präsentiert.

In der Arbeit „Ohne Titel“ (1985, Abb. 4) geschieht die Beschäftigung und Gestaltung auf der Grundlage dieser Matten mit Malutensilien und anderen Dingen. Sie werden nicht als Werkzeug zu einer malerischen Komposition benutzt, sondern sie werden selbst in einem entsprechenden Arrangement gezeigt, nicht vergleichbar mit einem Stillleben und dennoch in der Anordnung fixiert und ganz still geworden. Nicht die Abbildung ist das Ziel, sondern so wie - und das fand bereits Erwähnung - der Tisch, die Tischplatte in ihrer ganz anderen Funktionalisierung zum Artefakt wird, so auch die versammelten Utensilien.

Die Akkumulationen von Roth sind im Gegensatz zu den ,Fallenbildern “von Daniel Spoerri (Abb. 5) nicht zufällig, sondern arrangierte Materialanhäufungen und keine Abfallprodukte und auch keine Fundstücke. Die Produktionsstätte, das Atelier und als relevanter Bestandteil der Tisch, die Tischplatte mit den vielen Facetten künstlerischen Tuns wird zu einem Kunstwerk, zu einer künstlerischen Installation und in der Ausstellung wird diese Konstellation inszeniert. Inszenierungen - die Inszenierung des künstlerischen Mikrokosmos zu repräsentativen Zwecken, so zeigt sich die barocke Malerei (Abb.1 und 2) - Inszenierung des künstlerischen Mikrokosmos auch im Werk von Dieter Roth (Abb. 3 und 4), wobei hier nicht die Repräsentation im Vordergrund steht, sondern die Präsentation der künstlerischen Arbeitswelt, die gleichzeitig das Atelier und die Ausstellung zeigt - Verlagerung des Wirkungsfeldes -, und anders als in einer malerischen Darstellung können die Betrachtenden hier nicht nur auf Distanz sehen, sondern gegebenenfalls - den Ausstellungsmodalitäten entsprechend - auch fühlen, riechen, schmecken, sehen und hören. Die „Große Tischruine“ (Abb. 3) von Dieter Roth lässt sich in dieser Hinsicht als ein „Fünf - Sinne Stillleben" bezeichnen.

Von einer auch nur annähernd gedachten Wiedergabe der Geschichte des Stilllebens ist dieser Text weit entfernt; dennoch ist der Tisch Träger, ,stummer Diener“ einer jeden Anordnung und Darstellung von Gegenständen, unter welcher speziellen Überschrift auch immer. Meist ist nur die Tischplatte des Trägers in Ausschnitten sichtbar, die Stillleben vergangener Epochen und Stile betreffend. Roth und auch Spoerri, um Aspekte seines Werkes wird es im Folgenden noch gehen, entfernen die Tischplatte von den tragenden Beinen und verschaffen ihr Autonomie als Tafel-Bild. Der gemalte Gegenstand erscheint selbst in 
der Installation oder Komposition. Insgesamt wird das Atelier selbst zum Kunstwerk. Die Arbeit an sich ist identisch mit der künstlerischen Handlung.

Die Abbildung als Form kreativen Tuns mit Augentäuschungen, mit dem Phänomen des so genannten „Quodlibet" ${ }^{\star 4}$ - Wie es gefällt - ist als historische Grundlage der Darstellung des Ateliers von Dieter Roth im Ausstellungskontext und der „Fallenbilder“ von Daniel Spoerri zu sehen.

\section{„Bronze(n) in alle Ewigkeit“ - Daniel Spoerri}

Das in der Horizontale stattfindende Ereignis und in gewisser Weise komponierte Ensemble, das zu einem Bild wird, ist als künstlerische Handlung aus dem Werk von Daniel Spoerri hinlänglich bekannt. Die „Fallenbilder“ sind Ergebnisse unterschiedlichster Zusammenkünfte Freunde bei einem gemeinsamen Essen beispielsweise -, und die Arrangements wurden von dem Künstler nach dem Prinzip des Zufalls an einem von ihm zu bestimmenden Punkt fixiert. Die Gegenstände, die aus unbestimmt bestimmten Situationen und Begebenheiten erzählen, werden auf diese Weise für die Nachwelt inszeniert. Die Tischplatten verlieren ihre tragfähigen Beine und werden zu Tafelbildern. Und darin sind sie den ,Mattenbildern “ von Dieter Roth verwandt. Stellvertretend ist in diesem Text der „Mittagstisch in alle Ewigkeit “ (Abb. 5), 1994 datiert, abgebildet. ${ }^{5}$

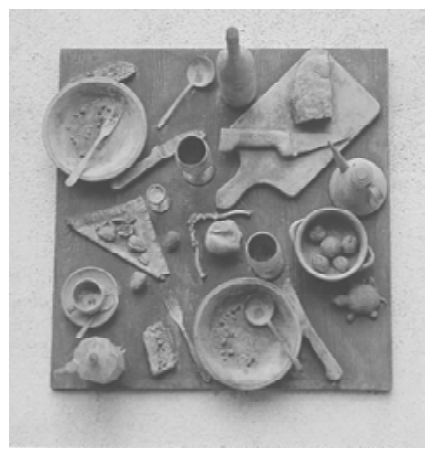

Abb. 5: Daniel Spoerri „Mittagstisch in alle Ewigkeit"

4 Zum Beispiel Cornelius Biltius, Quodlibet, 1684, 54,7 x 47,8 cm, Bonn, Rheinisches Landesmuseum.

5 An dieser Stelle sei auf den Beitrag von Gabriele Huber verwiesen, die sich intensiv mit dem ,Bronze - Atelier' von Daniel Spoerri auseinandersetzt. Dies sei als Vermerk und Querverweis dienlich, denn die Verwandtschaft zwischen dem „Bronze-Atelier" und dem „Mittagstisch in alle Ewigkeit“ (Abb. 5) ist offensichtlich. 
Alle „Fallenbilder“ dieses Künstlers konservieren Momente für die Ewigkeit, halten ganz bildlich alltägliche Gegebenheiten fest - nichts anderes ist in den so genannten Stilleben das Anliegen - und in dem „Mittagstisch in alle Ewigkeit“ (Abb. 5) potenziert sich diese Intention, indem der Künstler das Arrangement in Bronze gießt und somit zu einem Denkmal erhebt.

\section{Dieter Roth und Joseph Beuys - Die ,Ruine‘ und die ,Barraque‘ im Dialog}

Nicht nur der Titel der Installation von Dieter Roth „Große Tischruine“ (Abb. 3) veranlasst einen Vergleich, einen Dialog mit einer anderen Installation, mit der „Barraque D’Dull Odde“(Abb. 6) von Joseph Beuys.

Abb. 6: Joseph Beuys ,Barraque D’Dull Odde"

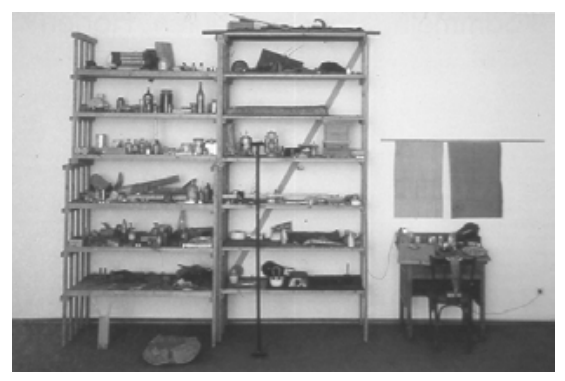

Hier Ruine, dort Barraque. Roth nimmt den Tisch, den wesentlichen Teilhaber an der Installation sogar mit in den Bildtitel und kombiniert den tragenden Teil mit dem Rudimentären, Fragmentarischen. Nimmt man den Begriff der „Ruine“, so entstehen ganz verschiedene Assoziationen: die künstlich errichtete Ruine in der Zeit der Romantik oder die durch Zerstörung, Feuer, Krieg etc. so gewordene Architektur. So oder so verbindet sich mit dem Begriff der "Ruine“ etwas Gebautes, Gebildetes, Materielles - und nicht das Atelier eines Künstlers. Ähnliches lässt sich bezüglich der Assoziationen zu der „Barraque“ denken. Beziehen sich die jeweiligen Titel auf das Verlassen, das Zurücklassen?

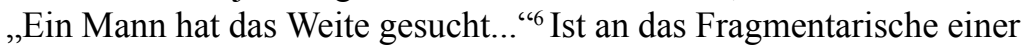
künstlerischen Handlung im Kontext von Welt und entsprechenden Fragestellungen gedacht?

Wie dem auch sei - sowohl für eine „Ruine“ als auch für eine „Barraque“ sind die jeweiligen Installationen, Inszenierungen äußerst komplex. Grundsätzlich stellt sich die Frage nach den äußeren wie in-

6 Gerhard Storck: Ein Mann hat das Weite gesucht, Transit. Plastische Arbeiten und Zeichnungen 1947-1985. 3 Bde., Krefeld 1991, S. 13. 
neren Gegebenheiten eines Künstlerateliers in der zweiten Hälfte des 20. Jahrhunderts. Nicht das konventionelle Maleratelier oder das strenge Bildhaueratelier scheint von Bedeutung, sondern der Gedanke an ein Laboratorium, an eine geistige Versuchs- und Auseinandersetzungsstätte. Die reine Abbildung der Welt, malerisch, zeichnerisch oder plastisch ist seit der Erfindung der Fotografie in den Hintergrund künstlerischer Interessen gerückt. Die Auseinandersetzung mit einer von Technik und Materialvielfalt bestimmten Welt ist in das Zentrum der Aufmerksamkeit gerückt, und es betrifft nicht nur die Werke selbst, sondern auch die Prozesse ihrer Entstehung.

Hinzu kommt der wesentliche Aspekt, dass insgesamt die Requisiten der Kunst immer profaner werden, in dem Anliegen, den Alltag so authentisch wie möglich zu spiegeln. Hier sei nur kurz an die bereits am Anfang des Jahrhunderts durch Marcel Duchamp und Man Ray initiierten Werke, die ohne Eingriffe und Veränderungen, Alltägliches zur Kunst erklärt haben. Sowohl in der Arbeit von Roth als auch in der von Beuys spielen alltägliche Gegenstände die eigentliche Rolle, die Hauptrolle.

Im Hinblick auf die ,, Große Tischruine " sei an den Anfang zurückgekehrt. Tische, Staffeleien und andere für die Erstellung künstlerischer Arbeiten wichtige Utensilien versammeln sich in einem Raum. Im Zuge der Arbeit in und mit diesen Gegenständen, entwickelt der Künstler die Idee, diese Gegenstände, die aufgrund ihrer Wertigkeit zusammengetragen und nicht ihren Weg in neue Zusammenhänge gegangen sind. Diese Dinge finden sich nun doch in ihrer Zurückgelassenheit als Gesamtheit an einer Stätte der Auseinandersetzung wieder, in der sich die Betrachtenden bewegen und sich damit auseinandersetzen können.Tische, die Protagonisten dieser und jener Arbeit, werden als stumme Diener bestückt, benutzt und letztlich so belassen. Ein Tisch jedoch wird von Roth hochkant gestellt, wird damit zum Tafelbild. Die Arbeitsfläche weist Farbstreifen auf, deren Herkunft sich in Form von festgeklebten Farbdosen und Gläsern ebenfalls auf der Tischfläche befindet. Eine aus dem Bürobereich stammende praktikable Klemmleuchte beleuchtet das Ganze von oben - ganz im Sinne einer Galerieleuchte, - ein ausgestelltes Bild wird in das - rechte Licht gesetzt. Der Tisch ist zur Ruhe gekommen, die Spuren der auf ihm verrichteten Arbeit haben ihn nun selbst zu einem Bild werden lassen. Andere Tische in dieser, Ruine' tragen verantwortlich das auf ihnen Abgestellte; teilweise sind sie so voll, dass sie in dieser Funktion einem Regal gleichen, teilweise bieten sie noch so viel Platz, dass man sich an ihnen aufhalten könnte. Die Ruine stellt eine Mischung dar aus dem nicht mehr gebraucht werden der dort belassenen Erinnerungs- 
stücke zum einen, und aus dem Denkmalcharakter für die dort mit diesen Stücken verrichtete Arbeit zum anderen.

Zwischen lebendig und tot manifestiert sich hier das Einfrieren eines über Jahre, Jahrzehnte dauernden Prozesses. Und im Grunde wird hier alles zum Tisch, selbst die Regale und zum Teil der Boden, auf dem sie, die Ruine, errichtet ist. In der Fülle alles dessen ist hier kein Platz mehr für einen neuen, anderen kreativen Prozess, die Requisiten selbst werden zum Prozess.

In der ,Barraque D'Dull Odde“ von Joseph Beuys, installiert in Krefeld im Kaiser-Wilhelm-Museum, ist ein kleiner Tisch Bestandteil, neben dem Zaun, zwei Regalen und vielen Details. Das reflektierende forschende Denken dokumentiert sich in beiden Arbeiten, in jeder auf ihre Weise. Aus dem inneren Prozess des Denkens entsteht der äußere Prozess des Forschens, Schreibens und Dokumentierens. In der „Barraque“ von Beuys gibt es Material und Werkzeuge. In der Installation fehlt der Mensch, das „Laboratorium“ ist verlassen. Es ist der Arbeitsplatz des Künstlers selbst, wie könnte er zugegen sein?

Das verlassene Denklabor, in dem aber bereits etwas erfunden und erdacht worden ist - die immer wieder aufleuchtende rote Lampe auf dem Tisch bringt Licht in die Dunkelheit und eröffnet möglicherweise die Hoffnung, dass es kein endgültiges Verlassen ist. Die Betrachtenden bleiben außen vor - sie stehen vor einem Zaun aus Maschendraht und Dachlatten - die Anmutung eines Kellerraumes, eines Abstellraumes drängt sich auf.

„Es gibt in der dramatischen Kunst eine aufschlussreiche Parallele zur ,Barraque D'Dull Odde'. Sie ist deshalb so aufschlussreich, weil in dem Bühnenstück an einer Stelle nicht nur eine vergleichbare Raumsituation beschrieben wird, sondern auch jener Wissenschaftler/Künstlertyp selbst auftritt und zu Worte kommt, der in der ,Barraque' vergraben gelebt haben muss, jetzt aber allein als Abwesender die Szene beherrscht."

7 Vgl. Gerhard Storck: Ein Mann hat das Weite gesucht, S. 13. „Bilde mir nicht ein, was Rechts zu wissen, Bilde mir nicht ein, ich könnte was lehren, Den Menschen zu bessern und zu bekehren. [...] Dass ich nicht mehr mit saurem Schweiß Zu sagen brauche, was ich nicht weiß; Dass ich erkenne, was die Welt Im Innersten zusammenhält, Schau' alle Willenskraft und Samen Und tu' nichtehr in Worten kramen. [...] O Sähst du, voller Mondenschein, zum letzten Mal auf meine Pein, Den ich so manche Mitternacht An diesem Pult herangewacht: Dann über Büchern und Papier, Trüb'selger Freund, erscheinst du mir! [...] Weh! Steck' ich in dem Kerker noch? Verfluchtes dumpfes Mauseloch, Wo selbst das liebe Himmelslicht Trüb durch gemalte Scheiben bricht! Beschränkt in diesem Bücherhauf', Den Würme nagen, Staub bedeckt, Den bis ans hohe Gewölb' hinauf Ein angeraucht Papier umsteckt; Mit Gläsern, Büchsen ringsum stellt, Mit Instrumenten vollgepfropft, Urväter - Hausrat drein gestopft Das ist deine Welt! Das heißt eine Welt!“" 
Die sichtbaren und die denkbaren Spuren in der Installation von Joseph Beuys sind unter dem Aspekt des Erinnerns, der Erinnerung relevant. Sowohl die vielen Gegenstände in den Regalen, als auch der Schreibtisch selbst sind damit bedeckt.

„Der Schreibtisch. Ausgrabungsort und Depot der Erinnerungen "- so ist ein Artikel von Annegret Pelz überschrieben, der sich mit genau diesem speziellen Tisch beschäftigt. ${ }^{8}$ In diesem Aufsatz beschäftigt sich die Autorin mit verschiedenen Dichterinnen, Schriftstellerinnen und deren jeweiligen Beziehungen zu ihren Schreibtischen. Der eigene lebensgeschichtliche Rückblick wird beispielsweise mit dem Schreibtisch in Verbindung gebracht. Der Schreibtisch als „Produktionsort und Ausgangspunkt der Erinnerungen“ und dabei ist „,das Möbel nicht nur Diener ihrer Tätigkeit, sondern in seinen räumlichen Dimensionen zugleich ihr Medium und Gedächtnisort". Jeder Mensch, der an einem Schreibtisch arbeitet, wird den Vorgang kennen, unter der Absicht aufzuräumen, immer wieder mit Fundstücken konfrontiert zu sein, die eigentlich keinen eigenen Platz haben, aber dennoch einen Platz beanspruchen. Und so werden sie wieder und wieder in die Hand genommen und halten somit entsprechende Begebenheiten, vielleicht bereits Erinnerungen wach. In diesem Zusammenhang findet der Begriff „Schreibtischreise“" Einzug in die Gedanken. „Sie ist vom ,Zufall“ der Entdeckungen und Erfindungen am jeweiligen Ort bestimmt, die der Sammlerin bei der Sichtung ihrer Schätze von außen zustoßen."

Wer kennt nicht den Moment größter Ergriffenheit im Anblick des Schreibtisches beispielsweise von J.W. von Goethe. Die Wahrnehmung des Gegenstandes, an dem viele der bereits gelesenen Zeilen entstanden sind, hat Bedeutung. Die Frage nach der Inszenierung solcher Gedenkstätten findet im Rahmen dieser Publikation am anderen Ort gebührende Beachtung.

\section{Das Atelier als performative Ausstellung - Ivan Kožarić}

Eine Pointierung der Vorgehensweise, ein Atelier auszustellen, findet sich in der Arbeit des kroatischen Künstlers Ivan Kožarić. So geschehen auf der letzten Documenta, allerdings von dem Künstler in der Absicht, während der 100 Tage in dem ausgestellten Atelier auch zu arbei-

8 Annegret Pelz: „Der Schreibtisch. Ausgrabungsort und Depot der Erinnerungen“, in: Magdalene Heuser (Hg.): Autobiographien von Frauen. Beiträge zu ihrer Geschichte, Tübingen 1996, S. 233-247.

9 Der Begriff der Schreibtischreise stammt von Sophie von La Roche, zit. nach: Annegret Pelz: Der Schreibtisch, S. 235.

10 Vgl. Annegret Pelz: Der Schreibtisch, S. 235. 
ten. Dies wurde von der Leitung der Ausstellung jedoch untersagt. So wurde auch dieses Atelier zu einer Inszenierung in Abwesenheit Anwesender, von daher gedanklich in der Nähe zu der „Großen Tischruine“ von Dieter Roth anzusiedeln.

„Die Installation ,Atelier Kožarić‘ kritisiert nicht nur eloquent das Konzept retrospektiver Ausstellungen allgemein, sondern auch die statische Natur von Kunstwerken. In Kozariæs Welt ist ein Objekt nichts weiter als ein Vorwand für noch ein weiteres, dies wiederum für das nächste und so fort. ,Atelier Kožarićc ist Performance, ist Installation und Labor in einem und verkörpert anschaulich die Arbeit eines Künstlers, dessen Werk in kontinuierlichem Experimentieren besteht." ${ }^{\prime 11}$
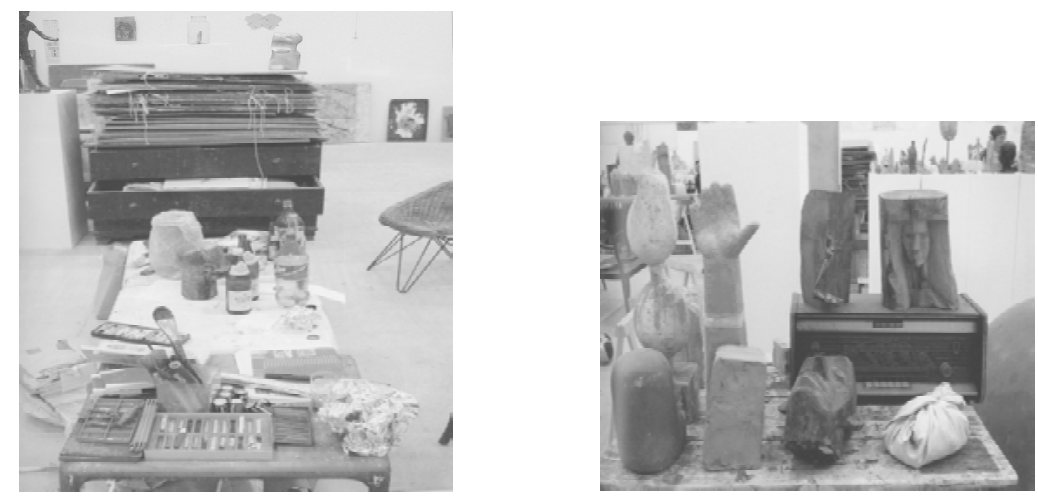

Abb. 7 und 8: Ivan Kožarić , Atelier Kožarić“

Der Vergleich mit einem Labor, ebenso das Prozesshafte der Arbeit, das Performative der Ausstellung, die Benennung als Installation und auch der Begriff des Experimentierens im Kontext künstlerischer Schaffensweisen greift als Beschreibung zeitgenössischer, respektive auch bereits älterer Kunstwerke - die ,, Große Tischruine “ begann Dieter Roth in den siebziger Jahren des 20. Jahrhunderts. Im Gegensatz zu der ,, Großen Tischruine“ und auch zu der „Barraque ... “ wollte der Künstler während der Ausstellung anwesend sein und in seinem Atelier, gleichsam in situ, arbeiten. Das Atelier auszustellen ohne darin arbeiten zu können, zu dürfen, bedeutet einen deutlichen Einschnitt für den Künstler. So war er abwesend, wie Roth und Beuys auch, jedoch unbeabsichtigt.

11 Carlos Basualdo, in: Documenta 11_Plattform 5. Kurzführer, Kassel 2002, S. 142. 
Interessant der Aspekt, dass die-Kunst Rezipienten oftmals nur mit Hilfe von dokumentarischen Fotografien und Filmen Einblick in die Werkstatt, das Atelier, die Produktionsstätte erhalten. Im Rahmen der Documenta11 beispielsweise konnten sie unterschiedliche Formen zeitgemäßer Inszenierungen der Orte im Original betrachten und erleben.

\section{Victor Grippo: „Tische zum Arbeiten und Nachdenken“}

Im Rahmen der Documenta11 waren auch Tische des argentinischen Künstlers Victor Grippo zu sehen (Abb. 9 und 10). Die Arbeit besteht aus sieben in Havanna gekauften Tischen, von denen mehrere während der ersten kubanischen Alphabetisierungsmaßnahme von 1959 als Schulplatte gedient haben. Auf die Tische hat Victor Grippo ausgewählte Texte argentinischer Autoren geschrieben. Die Installation beruft sich auf die Arbeit „Tabla“ (Tisch 1978) - ein Tisch mit einem Text des Künstlers selbst über die profane Helligkeit von Räumen, die von mehreren Generationen benutzt werden, um darin zu essen, zu lernen, zu denken, zu arbeiten und zu feiern. In den Räumen, an den Tischen - so erklärt sich auch der Werktitel dieser Installation „Tische zum Arbeiten und Nachdenken“.
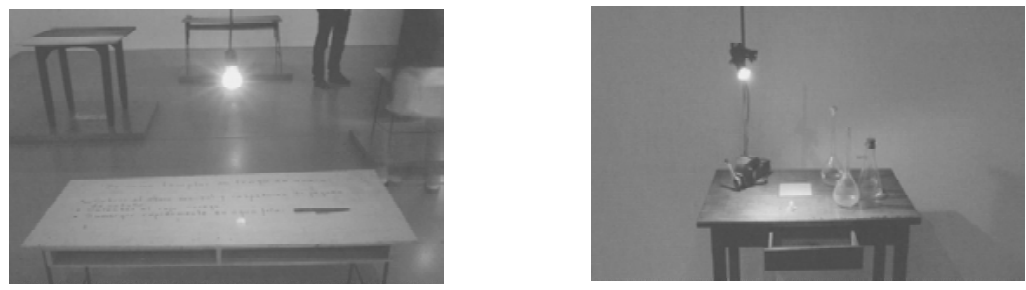

Abb. 9 und 10: Victor Grippa „,Tische zum Arbeiten und Nachdenken“

„Über diesem Tisch, Bruder von unendlich vielen anderen - vom Menschen gebaut, Ort des Zusammentreffens, des Nachdenkens, der Arbeit - wurde das Brot gebrochen, soweit es welches gab; die Kinder machten ihre Aufgaben, man weinte, es wurden Bücher gelesen, man teilte die Freuden." ${ }^{\prime 13}$

Die paradigmatische Figur des Künstlers taucht in Verbindung mit der des Handwerkers - mit Bäckern oder Tischlern - in den Plastiken und Installationen von Grippo immer wieder auf. Er sieht den heutigen Künstler, der das Individuum und die Gesellschaft umzugestalten sucht, als

12 Victor Grippo, Documenta11_Plattform 5 [Übersetzung aus dem Spanischen unter http://www.universes.de/car/documenta/11/frid/d-grippo-zoom3.htm]. 
moderne Inkarnation des mittelalterlichen Alchimisten. Auf der Suche nach Gold, auf der Suche nach etwas, das wertvoll ist und zu einer adäquaten Ausdrucksfindung bedarf es mehr als traditioneller Formen und Inhalte. Auch wenn die Installation von Victor Grippo nicht ein Künstleratelier meint, so ist seine Inszenierung des Ortes mit den Tischen, mit dem Licht - Glühbirnen beleuchten die Tische - und den Requisiten dennoch relevant in diesem Kontext. Seine Installation bezieht alltägliche Dinge mit ein, die die Schnittstelle zwischen den verschiedenen Bereichen deutlich machen. Die Werkbank des Tischlers ist unter Plexiglas gestellt; so wird dieser alltägliche, der Arbeit dienende Gegenstand a priori zu einem Ausstellungsstück, zu einer Skulptur, die geschützt werden muss.

In dem ausgewählten Detail (vgl. Abb. 9) wird ein Tisch mit einer geöffneten Schublade sichtbar. Auf der Tischplatte stehen eine Lampe und verschiedene Reagenzgefäße. Papier und Stift vervollständigen das Ensemble des Forschers. Und nicht zuletzt das Wort, die Schrift, die sich auf nahezu jedem Tisch befindet, ergänzt die Inszenierung, das dramaturgische Konzept. Die so vermittelten Inhalte ergänzen die Präsenz der Tische, an denen sowohl manuelle, wie auch geistige Tätigkeiten stattfinden. An einem Tisch ebenso wie in einem Atelier.

\section{Resümee}

Die angeführten Beispiele aus der Geschichte der Kunst zeigen Wirkungsstätten, die traditionelle Künstler-Persönlichkeiten zeigen. Die angeführten Beispiele von de Bailleur bis Velázquez beschreiben Maler und keine bildhauerisch tätigen Künstler. Sie verstehen sich als stellvertretende Beispiele. Die Wiedergabe der Wirklichkeit, die naturalistische Abbildung von Weltteilen, Aspekten von Welt - die Rede war von dem Mikrokosmos im Atelier - diente auch repräsentativen Zwekken. Das Gemälde von Cornelis de Bailleur (Abb. 1) setzt dem künstlerischen Mikrokosmos der damaligen Zeit ein Denkmal. ,Las Meminas “ von Velázquez setzt dem höfischen Leben und ihren Mitgliedern ebenfalls ein Denkmal - der Künstler gehört zu dem spanischen Hof ebenso wie die Bediensteten. Das Werk, die Auftragsarbeit entsteht im Atelier oder in situ. Für den ,Ort der Inszenierung ‘ ist der Raum in dem Gemälde des Spaniers beispielhaft.

In der Folge, wohl wissend, dass ein großer zeitlicher Sprung notwendig ist, wird das Atelier, nun nicht mehr Wirkungsstätte allein, selbst zu einem Gegenstand der Ausstellung. Nicht mehr das Gemälde, das das Atelier zeigt, ist Teil der Ausstellung, sondern der Raum selbst wird gezeigt. Der Künstler, die Künstlerin ist nicht anwesend - eine 
Ausnahme wäre die Präsenz von Ivan Kožarić im Rahmen der Documenta11 gewesen. In dem Ort der Inszenierung, dem zu einem Bild gewordenen Atelier oder dem zu einem Bild gewordenen künstlerischen Prozess ist der Tätige anwesend, sein Porträt sogar Bestandteil der Komposition. In der Inszenierung des Ortes fehlt der Protagonist, vorhanden sind die Spuren seiner Tätigkeiten, die Farben und Pinsel werden selbst zu einem Bild, die Tischplatte ist nicht mehr stummer Diener, sondern Bildgrund, Projektionsfläche. Die ausgestellten Requisiten geben Zeugnis von den vielfältigen künstlerischen Prozessen, von den inzwischen ganz anderen Medien, die notwendig, wenn nicht sogar Bedingung sind.

Der Tisch, der in allen aufgezeigten Beispielen eine tragende Rolle einnimmt, ist die Metapher für die Schnittstelle zwischen dem Denken und dem Handeln, dem Forschen und Planen der letztendlich entstehenden künstlerischen Arbeit. Sowohl die Orte der Inszenierungen, wie auch die Inszenierungen der Orte beinhalten eine weitere Dimension, die Qualität der Erinnerung. Allen Beispielen wohnt diese Qualität inne, nicht nur Wirkungsstätte, sondern auch Gedächtnisort zu sein. Der Tisch ist die Metapher für das Dasein, die Inszenierung des Ortes, der künstlerischen Wirkungsstätte ist die Metapher für die Kunstschaffenden. Auf diese Weise entstehen Gedächtnisorte in und für die Kunst.

\section{Literaturverzeichnis}

Basualdo, Carlos, in: Documenta11_Plattform 5. Kurzführer Kassel 2002.

Beuys, Joseph: „Barraque D’Dull Odde“, in: Transit. Plastische Arbeiten und Zeichnungen 1947-1985. 3 Bde., Krefeld 1991.

Grippo, Victor. Documenta11_Plattform 5. [Übersetzung aus dem Spanischen unter http://www.universes.de/car/documenta/11/frid/dgrippo-zoom3.htm].

Die Documenta11, in: Kunstforum International, Band 161, August Oktober 2002, S. 53-421.

Lange, Justus: „Galeriedarstellung mit Pictura Allegorie“, in: Pan und Syrinx. Eine erotische Jagd. Peter Paul Rubens, Pieter Brueghel und ihre Zeitgenossen. [Ausstell. Kat.], Kassel, Frankfurt/M. 2004.

Pelz, Annegret: „Der Schreibtisch. Ausgrabungsort und Depot der Erinnerungen", in: Magdalena Heuser (Hg.): Autobiographien von Frauen. Beiträge zu ihrer Geschichte, Tübingen 1996, S. 233 - 247.

Roth, Dieter, in: Das XX. Jahrhundert. Ein Jahrhundert Kunst in Deutschland. [Ausstell. Kat.], Berlin 1999.

Storck, Gerhard: „Ein Mann hat das Weite gesucht“, in: Transit. Plastische Arbeiten und Zeichnungen 1947-1985. 3 Bde., Krefeld 1991. 